\title{
LA NORMATIVA DE LA UE QUE PROHIBE DISCRIMINAR A LOS CLIENTES QUE COMPRAN EN LÍNEA: ¿CÓMO FUNCIONA EL COMERCIO ELECTRÓNICO TRANSFRONTERIZO?
}

\author{
Luis González Vaqué ${ }^{1}$ \\ "International Association of Consumer Law (IACL)" \\ Ex-Consejero de la Dirección General de Mercado Interior de la Comisión Europea
}

Resumen: El Reglamento (UE) 2018/302 relativo al bloqueo geográfico prohíbe la discriminación injustificada de los clientes que compran productos o servicios. Garantiza que la nacionalidad, el lugar de residencia o el lugar de establecimiento de los clientes no puedan utilizarse para denegar el acceso a una tienda en línea (por ejemplo, de productos electrónicos o de prendas de vestir) o a un servicio prestado en línea y consumido fuera de línea en la Unión Europea (por ejemplo, el alquiler de automóviles), también cuando esta discriminación esté relacionada con los medios de pago. La evaluación de su aplicación hasta la fecha es moderadamente optimista.

Palabras clave: Mercado interior, Comercio en línea, Bloqueo geográfico, Discriminación de los clientes, Protección de los consumidores.

Title: The EU rules that forbid discriminate customers who buy online: how does the cross-border electronic trade work?

\begin{abstract}
The Geo-blocking Regulation (EU) 2018/302 outlaws unjustified discrimination of customers buying goods or services. It ensures that customers' nationality, place of residence or place of establishment cannot be used to refuse access to an online shop (e.g. for electronics or clothing) or to a service provided online and consumed off-line (e.g. car rental) in the EU, including where this discrimination is related to means of payment. The assessment of its implementation to date is moderately optimistic.
\end{abstract}

Key words: Internal market, eCommerce, Geo-blocking, Customer discrimination, Consumer protection.

\footnotetext{
1 Dirección electrónica: gonzalu70@outlook.es
} 
Sumario: 1. Introducción: el Reglamento (UE) 2018/302 sobre el bloqueo geográfico injustificado. 2. El primer Informe de la Comisión europea sobre el Reglamento relativo al bloqueo geográfico. 3. Conclusiones

\section{Introducción: el Reglamento (UE) $2018 / 302$ sobre el bloqueo geográfico injustificado}

El Reglamento (UE) $2018 / 302^{2}$ tiene por objeto impedir las discriminaciones injustificadas en las ventas en línea por razón de nacionalidad, lugar de residencia o lugar de establecimiento de los clientes en el mercado interior. La prohibición del bloqueo geográfico es un elemento importante de la estrategia del Mercado único digital $\mathbf{l}^{3}$.

En efecto, su objetivo principal es contribuir al buen funcionamiento del Mercado interior de la Unión Europea (UE) impidiendo el bloqueo geográfico injustificado ${ }^{4}$ y otras formas de discriminación basada, directa o indirectamente, en la nacionalidad, el lugar de residencia o el lugar de establecimiento de los clientes, en las transacciones en línea y fuera de línea, dentro de la UE.

Aunque no obliga a los comerciantes a realizar entregas transfronterizas (si el comerciante todavía no presta este servicio) ni a la armonización de los precios, sí prohíbe la discriminación entre los clientes de la UE en cuanto al acceso a los bienes y servicios por razón de la nacionalidad, el lugar de residencia o el lugar de establecimiento, cuando el cliente extranjero acepta las condiciones aplicadas a escala nacional (es decir, el derecho a comprar como un cliente local).

El Reglamento (UE) 2018/302 entró en vigor en todos los Estados miembros de la UE el 23 de marzo de 2018 y es aplicable desde el 3 de diciembre de 2018.

El objetivo del presente artículo es doble: por un lado, permitir un mejor conocimiento de su contenido pues pensamos que por su falta de visibilidad ${ }^{5}$ no ha permitido que

\footnotetext{
2 Reglamento (UE) 2018/302 del Parlamento Europeo y del Consejo, de 28 de febrero de 2018, sobre medidas destinadas a impedir el bloqueo geográfico injustificado y otras formas de discriminación por razón de la nacionalidad, del lugar de residencia o del lugar de establecimiento de los clientes en el mercado interior y por el que se modifican los Reglamentos (CE) n. ${ }^{\circ} 2006 / 2004$ y (UE) 2017/2394 y la Directiva 2009/22/CE (DO L 60I de 2.3.2018, p. 1).

3 Véanse sobre este tema: MARIA LORENA FLÓREZ ROJAS (2018). Are online consumers protected from geo-blocking practices within the European Union?: International Journal of Law and Information Technology (26 - 2), 119-141; y GIUSEPPE MAZZIOTTI (2015). Is Geo-Blocking a Real Cause for Concern in Europe?: EUI Department of Law Research Paper No. 2015/43, 23 págs.: https://papers.ssrn.com/sol3/papers.cfm?abstract_id=2728675

${ }^{4}$ Véase: https://ec.europa.eu/digital-single-market/en/geo-blocking

5 Véase sobre la importancia de la visibilidad de las normativas legales para su efectiva aplicación: A. MAYORAL (2020). La visibilidad de la legislación: alcance e intereses en juego: BoDiA/Co (38) 11-20.
} 
los consumidores hayan obtenido una efectiva protección contra la discriminación en la "economía digital"; además, trataremos de resumir, en base a la documentación disponible cómo ha funcionado en las operaciones transfronterizas en línea la aplicación de la prohibición establecida en el citado Reglamento (UE) 2018/302.

Por ambas razones, nos parece oportuno, antes de seguir adelante, exponer mediante un pequeño resumen de la disposición en cuestión sus elementos básicos.

En este contexto, vale la pena reproducir textualmente sus dos primeros considerandos:

«(1) Para lograr el pleno potencial del mercado interior en tanto que espacio sin fronteras interiores en el que, entre otras libertades, se garantice la libre circulación de mercancías y la libre prestación de servicios, no basta con suprimir las barreras entre los Estados miembros creadas por los propios Estados. Esa supresión puede verse socavada por obstáculos creados por particulares y que son incompatibles con las libertades del mercado interior. Ello sucede cuando los comerciantes que ejercen su actividad en un Estado miembro bloquean o limitan el acceso a sus interfaces en línea, tales como sitios web y aplicaciones, a clientes de otros Estados miembros que desean realizar transacciones transfronterizas (práctica conocida como 'bloqueo geográfico'). También sucede cuando determinados comerciantes aplican a esos clientes de otros Estados miembros condiciones generales de acceso diferentes a sus productos y servicios, tanto en línea como fuera de línea. Si bien ese trato diferente podría, en algunos casos, estar justificado objetivamente, en otros casos, algunas prácticas de los comerciantes impiden o limitan el acceso a los productos o servicios a los clientes que desean realizar transacciones transfronterizas, o algunos comerciantes aplican, a este respecto, condiciones generales de acceso diferentes que no se justifican objetivamente.

(2) Existen diversas razones subyacentes por las que empresas, y en particular las microempresas y las pequeñas y medianas empresas (pymes), aplican condiciones generales de acceso diferentes. En muchos casos, los entornos jurídicos divergentes, la incertidumbre jurídica que ello implica, los riesgos asociados a la legislación aplicable en materia de protección de los consumidores, la legislación en materia de medio ambiente o etiquetado, las cuestiones tributarias y fiscales, los gastos de entrega o los requisitos lingüísticos, contribuyen a que los comerciantes sean reacios a entablar relaciones comerciales con los clientes de otros Estados miembros. En otros casos, los comerciantes segmentan artificialmente el mercado interior según las fronteras interiores y dificultan la libre circulación de mercancías y servicios, lo que limita los derechos de los clientes y les impide disfrutar de posibilidades de elección más amplias y unas condiciones óptimas. Estas prácticas 
discriminatorias son un factor importante que contribuye al nivel relativamente bajo de transacciones transfronterizas en la Unión, incluso en el sector del comercio electrónico, lo que impide alcanzar el pleno potencial de crecimiento del mercado interior. Por lo tanto, el presente Reglamento debe determinar las situaciones en que no puede haber ninguna justificación para un trato diferenciado, aportando así claridad y seguridad jurídica a cuantos participan en las transacciones transfronterizas, y garantizando la aplicación y el cumplimiento efectivo de normas contra la discriminación en todo el mercado interior. Eliminar el bloqueo geográfico injustificado y otras formas de discriminación por razón de la nacionalidad, del lugar de residencia o del lugar de establecimiento de los clientes podría impulsar el crecimiento y aumentar las opciones de los consumidores en todo el mercado interior.»

Por lo que se refiere al ámbito de aplicación del Reglamento (UE) 2018/302, según lo previsto en su artículo 1, subrayaremos que no se aplica a:

- las situaciones puramente internas de los países de la UE (es decir, aquellas en las que todos los elementos pertinentes de la transacción se circunscriben al interior de un único Estado miembro);

- otros servicios como los servicios financieros, audiovisuales, de transporte, de atención sanitaria y sociales, de conformidad con el ámbito de aplicación de la Directiva 2006/123/CE relativa a los servicios en el mercado interior ${ }^{6}$;

- ni a los servicios vinculados a contenidos protegidos por derechos de autor, como los servicios de emisión de música en continuo, los libros electrónicos, los programas informáticos y los videojuegos (exclusión pendiente de revisión).

Vale la pena añadir que el Reglamento prohíbe el bloqueo del acceso a sitios web y el reencaminamiento sin el consentimiento previo del cliente (incluso si el cliente manifiesta su consentimiento al reencaminamiento, la versión original visitada debe permanecer accesible). Cuando el bloqueo, la limitación de acceso o el reencaminamiento estén justificados por el Derecho de la UE, o por el Ordenamiento jurídico nacional de conformidad con el Derecho de la UE, los comerciantes deben facilitar a los clientes una explicación clara.

Existen tres situaciones específicas en las que los comerciantes no pueden aplicar condiciones generales de acceso diferentes a sus productos o servicios, por motivos relacionados con la nacionalidad o con el lugar de residencia o de establecimiento del cliente, salvo que sea para el cumplimiento de lo dispuesto en la legislación de la UE, o del propio Derecho nacional de conformidad con el Derecho de la UE:

\footnotetext{
6 Directiva 2006/123/CE del Parlamento Europeo y del Consejo, de 12 de diciembre de 2006, relativa a los servicios en el mercado interior (DO L 376 de 27.12.2006, p. 36).
} 
- la venta de productos que se entreguen en una zona donde preste servicio el comerciante (o sean recogidos por el cliente);

- la venta de servicios que se presten por vía electrónica; y

- la venta de servicios que se presten en un lugar físico específico.

Nuestro resumen no estaría completo si dejáramos de mencionar que los comerciantes son libres de aceptar los medios de pago y las marcas de tarjetas que deseen. No obstante, no se les permite discriminar en lo que respecta a los mismos medios de pago o marcas por motivos relacionados con:

- la nacionalidad, el lugar de residencia o de establecimiento del cliente;

- la ubicación de la cuenta de pago;

- el lugar de establecimiento del proveedor del servicio de pago; y

- el lugar de emisión del instrumento de pago.

Se considera que los comerciantes actúan de manera discriminatoria si rechazan un pago cuando:

- se realice a través de transacciones electrónicas mediante transferencia, adeudo domiciliado o pago con una tarjeta dentro de la misma marca y categoría;

- se realice en una moneda que el comerciante acepte; y

- se cumplan los requisitos de autenticación.

Sin embargo, los comerciantes pueden reclamar el abono de gastos por la utilización de un pago con tarjeta cuyas tasas de intercambio ${ }^{7}$ no estén contempladas por las reglas de la UE relativas a las tasas aplicadas a las operaciones de pago con tarjeta.

En el artículo 2 del Reglamento (UE) 2018/302 se incluyen diversas definiciones entre las que destacaremos:

- Bloqueo geográfico: una práctica discriminatoria de un comerciante para impedir a los clientes en línea acceder a los productos o servicios ofrecidos en un sitio web alojado en otro país y adquirir dichos productos o servicios, por

\footnotetext{
7 En el artículo 2.2 de del Reglamento relativo al bloqueo geográfico se establece que se entenderá tasa de intercambio «tal como se define en el artículo 2, punto 10, del Reglamento (UE) 2015/751 [del Parlamento Europeo y del Consejo, de 29 de abril de 2015, sobre las tasas de intercambio aplicadas a las operaciones de pago con tarjeta (DO L 123 de 19.5.2015, p. 1]».
} 
ejemplo, bloqueando el acceso transfronterizo a sitios web; denegando la posibilidad de realizar un pedido o de adquirir bienes o servicios cuando se accede a un sitio web desde otro país.

- Consumidor: toda persona física que actúe con fines ajenos a su actividad comercial, negocio, oficio o profesión.

- Cliente: un consumidor que sea nacional de un Estado miembro o que tenga su lugar de residencia en un Estado miembro, o una empresa que tenga su lugar de establecimiento en un Estado miembro, y que recibe un servicio o adquiere un producto, o tiene intención de hacerlo, en la Unión, únicamente para su uso final;

- Condiciones generales de acceso: todas las condiciones y otros datos, como los precios netos de venta, que regulen el acceso de los clientes a los productos o servicios ofrecidos a la venta por un comerciante, que se establezcan, se apliquen y se pongan a disposición del público en general por parte del comerciante o en su nombre y que se apliquen a falta de un acuerdo negociado individualmente entre el comerciante y el cliente;

- Productos: todo bien mueble tangible, excepto los bienes vendidos por la autoridad judicial tras un embargo u otro procedimiento;

- Interfaz en línea: cualquier tipo de programa informático, incluidos los sitios web o parte de ellos y las aplicaciones, incluidas las aplicaciones móviles, utilizado por un comerciante o en nombre de este, que sirva para dar a los clientes acceso a los productos o servicios del comerciante con vistas a iniciar una operación con respecto a dichos productos o servicios;

- Servicio: cualquier actividad económica por cuenta propia, prestada normalmente a cambio de una remuneración, contemplada en el artículo 57 del TFUE;

- Comerciante: toda persona física o jurídica, ya sea privada o pública, que actúe, incluso a través de otra persona que actúe en su nombre o a su cargo, con fines relacionados con su actividad comercial, negocio, oficio o profesión.

Por su parte, el artículo 6 se refiere a los siguientes conceptos:

- Ventas activas: en el contexto de los acuerdos de distribución exclusiva, las ventas realizadas mediante aproximación activa a clientes individuales en el territorio exclusivo de otro distribuidor.

- Ventas pasivas: ventas que atienden a solicitudes espontáneas de clientes situados en un territorio en el que no opere el comerciante. 


\section{El primer Informe de la Comisión europea sobre el Reglamento relativo al bloqueo geográfico}

La Comisión publicó recientemente un Informe haciendo un balance de la primera fase de aplicación del Reglamento relativo al bloqueo geográfico (en lo sucesivo el "Informe sobre el bloqueo geográfico"). Esta revisión temprana de la normativa comunitaria en cuestión se acordó en las negociaciones, en parte para evaluar la oportunidad de ampliar su ámbito de aplicación a otros servicios, como el contenido protegido por derechos de autor. Al mismo tiempo, la revisión finalizó en medio del estallido de la crisis de la COVID-19, por lo que los datos y el análisis de la revisión no pudieron tener en cuenta las posibles repercusiones de la pandemia.

Partiendo de la premisa de que «en un mercado único digital verdaderamente conectado, los consumidores esperan acceder a los productos y servicios sin restricciones más allá de las fronteras», en el Informe sobre el bloqueo geográfico, se subraya que tal principio implica que las situaciones en las que a un cliente que realiza compras transfronterizas se le impide finalizar el proceso de compra, o se le pide que pague con una tarjeta de débito o crédito de un determinado país deben ser prohibidas y sancionables: «el Reglamento tiene como objetivo aumentar las oportunidades de los consumidores y las empresas que realizan compras transfronterizas: los consumidores deben poder 'comprar como un local' en cualquier lugar de la UE» (ibidem).

Formalmente, el Informe sobre el bloqueo geográfico, que recoge las principales conclusiones de la Comisión sobre el primer período de aplicación del Reglamento, está estructurado en tres secciones principales:

- En la primera sección ["sección 2" (?) en el documento en cuestión] se examina la aplicación por parte de los Estados miembros y los comerciantes, así como la evolución de las expectativas de los consumidores.

- La sección 3 (sic en el Informe sobre el bloqueo geográfico) aborda las oportunidades y los desafíos que conlleva la modificación del ámbito de aplicación del Reglamento.

La sección 4 (idem) concluye con una enumeración de una serie de medidas de seguimiento.

Obviamente, en nuestro caso la "sección" que más nos interesa es la relativa a la aplicación del Reglamento (UE) 2018/302, y, en especial:

8 COM (2020) 766 final. 


\section{- La aplicación por los Estados miembros}

Recordaremos, como hace la Comisión, que, para los casos en que surja un litigio entre un consumidor y un comerciante, los Estados miembros tenían la obligación de designar, a más tardar el 3 de diciembre de 2018, a el/los organismo(s) encargado(s) de proporcionar asistencia práctica a los consumidores. También debían haber garantizado la disponibilidad de acciones de cesación, así como la cooperación en el seno de la red de cooperación en materia de protección de los consumidores $^{9}$ (red $\mathrm{CPC}^{10}$ ).

Los Estados miembros también estaban obligados a capacitar a los organismos competentes y establecer medidas para garantizar la ejecución adecuada y efectiva del Reglamento, de modo que pudieran adoptarse medidas contra los comerciantes que infringen las reglas de las que se trata.

Lamentablemente, el cumplimiento de estas obligaciones ha sido muy irregular:

«En general, los retrasos han frenado el proceso de aplicación por parte de los Estados miembros. En diciembre de 2018, solo seis Estados miembros habían adoptado y notificado las medidas y los organismos designados para la ejecución del Reglamento ${ }^{\mathbf{1 1}}$. A raíz de las actividades de estrecha supervisión y escrutinio llevadas a cabo por la Comisión, la mayoría de los Estados miembros adoptaron estas medidas durante la primavera de 2019. En julio de 2019, seis Estados miembros todavía no habían comunicado todas o parte de las medidas de ejecución aplicables en caso de infracción del Reglamento. La Comisión incoó procedimientos de infracción en estos casos» (Informe sobre el bloqueo geográfico).

Entre los Estados miembros que designaron los organismos pertinentes, la mayoría optó por capacitar a las autoridades responsables de proteger a los consumidores como organismos de ejecución, y a los Centros Europeos del Consumidor como organismos de asistencia ${ }^{\mathbf{1 2}}$. Sin embargo, existen divergencias considerables entre Estados miembros en cuanto a las sanciones aplicables y a menudo existe una gran diferencia entre las multas mínimas y máximas dentro de un Estado miembro ${ }^{13}$. En los casos de bloqueo geográfico entre empresas, los

\footnotetext{
9 Véase el Reglamento (UE) 2017/2394 del Parlamento Europeo y del Consejo, de 12 de diciembre de 2017, sobre la cooperación entre las autoridades nacionales responsables de la aplicación de la legislación en materia de protección de los consumidores y por el que se deroga el Reglamento (CE) n. ${ }^{0}$ 2006/2004 (DO L 345 de 27.12.2017, p. 1).

${ }^{10}$ La red CPC reúne a las autoridades encargadas de la aplicación de la legislación en materia de protección de los consumidores en la UE.

11 La cursiva es nuestra.

12 https://ec.europa.eu/digital-single-market/en/geo-blocking-digital-single-market\#Implementation

13 Desde unos pocos cientos de euros hasta multas superiores a 100000 euros o multas relacionadas con el volumen de negocios.
} 
Estados miembros se dividen casi por igual a la hora de seguir uno de los dos enfoques diferentes: bien mediante ejecución privada a través de los órganos jurisdiccionales únicamente; o bien a una combinación de ejecución privada y pública. Por consiguiente, la Comisión estima que habrá que seguir evaluando la eficacia de los sistemas de sanción y ejecución, en particular en los casos de empresa a empresa, también para evaluar la aplicación práctica y la eficacia de los recursos judiciales.

\section{- Expectativas y sensibilización de los consumidores}

Según la Comisión, el Reglamento (UE) 2018/302, que constituye una de las principales iniciativas relacionadas con el comercio electrónico de la Estrategia para el Mercado Único Digital, atrajo desde un primer momento una atención considerable por parte de los consumidores y sus organizaciones de representación: «uno de los motivos es que el Reglamento otorga directamente derechos específicos a los consumidores frente a los comerciantes transfronterizos» (Informe sobre el bloqueo geográfico).

Añadiremos que la Comisión también llevó a cabo actividades de sensibilización a través de diversos canales, mucho antes de la entrada en vigor del Reglamento que nos interesa. Una de estas acciones fue la publicación de documentos de preguntas y respuestas para aclarar las dudas tanto de los consumidores como de los comerciantes ${ }^{\mathbf{1 4}}$ (afortunadamente traducidas al español): «como resultado de ello, en febrero de 2019 , solo un par de meses después de su fecha de aplicación, aproximadamente el $50 \%$ de los consumidores de la UE ya conocían el Reglamento», lo que, en nuestra opinión, de ser cierto tampoco constituiría un verdadero éxito.

En su Informe sobre el bloqueo geográfico, la Comisión se ve obligada a aceptar las carencias del Reglamento (UE) 2018/302, pues muchas expectativas de los consumidores en lo que respecta a la denegación de la entrega transfronteriza, o a la ausencia de opciones de entrega para determinados países, especialmente por parte de comerciantes multinacionales se han visto frustradas: «el Reglamento no preveía obligaciones en estas esferas [por lo que] la Comisión propone que se siga sensibilizando sobre el Reglamento, al tiempo que se siguen vigilando las prácticas generales de comercialización de los comerciantes y las repercusiones de esas prácticas en las opciones de las que dispone el público en toda la UE» (ibidem).

\footnotetext{
14 Disponibles en https://ec.europa.eu/digital-single-market/en/news/geo-blocking-regulation-questionsand-answers
} 


\section{- Aplicación por los comerciantes}

Debido a la falta de visibilidad del Reglamento (UE) 2018/302, los resultados tampoco son especialmente favorables:

«... una encuesta realizada en 2019, que abarcaba alrededor de 9000 sitios web, mostró algunos efectos positivos iniciales del Reglamento en la mejora de la accesibilidad transfronteriza ${ }^{\mathbf{1 5}}$ de los sitios web de los comerciantes [...]. En comparación con [otra encuesta de] 2015, el reencaminamiento ${ }^{\mathbf{1 6}}$ o la denegación de acceso a los sitios web de comercio electrónico había disminuido $[\ldots]$, mientras que las restricciones que impedían totalmente el registro en los sitios web se redujeron a la mitad [...]. También han disminuido los cambios automáticos de los precios en el momento del registro. En general, el porcentaje de compras transfronterizas realizadas con éxito aumentó ligeramente en comparación con 2015 [...].

Sin embargo, más de uno de cada diez intentos transfronterizos de compras simuladas fracasó en la etapa de registro, y un número similar en la etapa de pago. Esto se debió normalmente a requisitos de residencia o de ubicación de los pagos (por ejemplo, solo se aceptaban tarjetas de débito emitidas localmente) [...]. Hay una necesidad permanente de hacer frente a estos obstáculos, que pueden: i) tener un carácter discriminatorio; ii) dar lugar a resultados sesgados; o iii) impedir el acceso a las ofertas y la comparación de las mismas. Para abordar estos problemas es necesario contar con organismos de ejecución y de asistencia plenamente operativos en todos los Estados miembros.

\section{$[\ldots]$}

El Reglamento no obliga a los comerciantes a realizar entregas transfronterizas cuando las condiciones aplicables no lo contemplen ${ }^{\mathbf{1 7}}$. Sin embargo, a menudo el proceso de compra se detiene en las últimas etapas porque el comerciante no realiza entregas en el país del comprador, de modo que cuando se solicitaba la entrega transfronteriza, solo uno de cada tres intentos de compra transfronteriza tuvo éxito en el reciente ejercicio de compra simulada ${ }^{\mathbf{1 8}}$.

\footnotetext{
15 La cursiva es nuestra.

16 Idem.

17 Por otra parte, si el comerciante, en sus condiciones generales, ya prevé la entrega transfronteriza en el país del cliente no debe discriminarse a ese cliente.

18 Esto concuerda con las cifras generales de Eurostat sobre el número de empresas que se dedican de manera activa a las ventas transfronterizas, que se mantuvieron estables en 2019 en comparación con 2017. Solo una minoría de empresas realiza ventas transfronterizas, incluso en el sector en línea (el $43 \%$ de las empresas que operan en línea venden en el ámbito transfronterizo, en comparación con el $9 \%$ del total de las empresas).
} 
Esto se debe principalmente a que siguen existiendo limitaciones de entrega en las compras transfronterizas. Estas restricciones todavía afectan a más del 50 $\%$ de los intentos de compra ${ }^{\mathbf{1 9}}$, incluso con comerciantes multinacionales que ofrecen diferentes versiones nacionales de sus sitios web.

Aunque en esta etapa el Reglamento no los aborda de manera directa, esos problemas pueden frustrar las expectativas de los consumidores de poder acceder a las ofertas en todo el mercado único europeo. Por otra parte, los comerciantes en línea siguen enfrentándose a obstáculos administrativos (como los requisitos de registro del IVA) y no tienen claros los requisitos de protección del consumidor que se aplican a las ventas transfronterizas. Por lo tanto, estas cuestiones pueden disuadir a los comerciantes de aumentar su implicación a escala transfronteriza ${ }^{20}$.

Esta información sugiere que se necesita un enfoque holístico para lograr todos los efectos previstos por el Reglamento ${ }^{21}$. Dicho planteamiento promovería un mayor acceso transfronterizo para los consumidores, al tiempo que facilitaría la implicación a escala transfronteriza de los comerciantes.» (Informe sobre el bloqueo geográfico).

Para no alargarnos más, no nos ocuparemos aquí de la sección relativa a la "Ampliación del ámbito de aplicación del Reglamento"; los lectores interesados en los diversos sectores [especial pero no exclusivamente los servicios prestados por vía electrónica que permiten acceder a contenido protegido por derechos de autor (música, libros electrónicos, juegos o programas informáticos, servicios audiovisuales, etcétera)] pueden consultar el Informe sobre el bloqueo geográfico: https://eur-lex.europa.eu/legalcontent/ES/TXT/HTML/?uri=CELEX:52020DC0766\&from =ES

\section{Conclusiones}

En este epígrafe entrelazaremos nuestras propias conclusiones con las "Acciones de seguimiento de la Comisión" que figuran al final del Informe sobre el bloqueo geográfico. Estas últimas se proponen de una forma en cierto sentido provisional porque la propia Comisión «... considera que es demasiado pronto para evaluar todos

\footnotetext{
19 Esta cifra del $50 \%$ incluye también los casos en que se notificaron problemas con la entrega surgidos durante el registro (no se acepta la dirección de entrega en la fase de registro).

20 Estos son los segundos y terceros obstáculos más importantes para los minoristas respectivamente (después de la recuperación de la deuda) y todavía afectan a una gran parte de las empresas (el $60 \%$ de las empresas citan los procedimientos del IVA como un obstáculo para la venta transfronteriza). Véase el documento de trabajo de los servicios de la Comisión titulado: «Business Journey on the Single Market: Practical Obstacles and Barriers» (Itinerario de las empresas en el mercado único: barreras y obstáculos prácticos) [SWD (2020) 54 final].

${ }^{21}$ La cursiva es nuestra.
} 
los efectos directos e indirectos de la ejecución del actual Reglamento y determinar qué modificaciones se requieren al respecto».

Por el contrario, en nuestra opinión no es demasiado pronto en aumentar la visibilidad de esta normativa e informar debidamente a los consumidores de los derechos que les reconoce el del Reglamento (UE) 2018/302 ${ }^{\mathbf{2 2}}$. Lamentamos la lentitud de la aplicación de dicho Reglamento y la discordancia de las medidas adoptadas en su caso, aunque, «...los efectos positivos iniciales que se recogen en el [Informe sobre el bloqueo geográfico] indican que existe potencial en un futuro cercano para obtener mejores resultados a lo largo del tiempo, en particular mediante la mejora de la ejecución en los Estados miembros ${ }^{23}$ »; y nos parece que la Comisión debería esforzarse más en fiscalizar sus actividades...

Además, sería conveniente fomentar toda posible sinergia con otras medidas del Mercado único digital para mejorar el acceso de los clientes a las ofertas. Quizás una refundición de todas las Directivas y Reglamentos aplicable en una única medida legislativa comunitaria sería deseable.

Coincidimos con la Comisión en que, «en lo que se refiere a la posibilidad de ampliar el alcance del Reglamento a los servicios prestados por vía electrónica que proporcionan acceso a contenidos protegidos por derechos de autor, es necesario proseguir la evaluación y el examen» (Informe sobre el bloqueo geográfico). Es cierto que el bloqueo geográfico en estos sectores tiene su origen en diferentes factores y dinámicas de mercado, pero no nos parece aceptable la ambigua conclusión de «... que la ampliación del ámbito de aplicación del Reglamento podría tener diferentes efectos en distintos ámbitos» (ibidem). En definitiva, «todo efecto beneficioso, en particular para los consumidores, depende en gran medida de las prácticas y planteamientos de concesión de licencias de derechos de autor, que merecen un análisis más pormenorizado. En esta situación se encuentra, en particular, el sector audiovisual, en el que, en efecto, es posible que exista potencial para lograr mejoras importantes en cuanto a la disponibilidad de contenido. Esto confirma la necesidad de examinar la cuestión del acceso de los consumidores a los contenidos, en particular a los contenidos audiovisuales, en el contexto más amplio de respaldo a la industria en su recuperación y transformación en el futuro plan de acción de la Comisión sobre los medios de comunicación y el sector audiovisual» (ibidem).

Resulta esperanzador que la Comisión tenga la intención de entablar «... un diálogo con las partes interesadas para determinar cómo mejorar la difusión de los

\footnotetext{
22 Por ejemplo, mediante una referencia obligatoria en la correspondiente página de Internet del comerciante.

${ }^{23}$ La cursiva es nuestra.
} 
contenidos audiovisuales en toda la UE y [que], al mismo tiempo, propondrá medidas

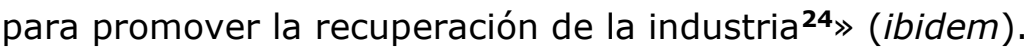

Opinamos también que es un acierto que la Comisión siga teniendo como prioridad la eliminación de las barreras existentes y el fin de la discriminación de los clientes. En el Informe sobre el bloqueo geográfico se proponen las siguientes medidas de seguimiento inmediato.

- En primer lugar, es importante garantizar que se aproveche todo el potencial del actual Reglamento. Ello requiere:

i) una aplicación completa por los Estados miembros;

ii) una mayor cooperación entre las autoridades competentes y la Comisión en el marco de la nueva red CPC; $y$

iii) más orientación sobre la aplicación del Reglamento y su relación con el marco más amplio de no discriminación de la Directiva de servicios.

-.En segundo lugar, es necesario que prosigan las actividades que sensibilizan a los comerciantes y los consumidores acerca de sus obligaciones y derechos. La Comisión debe participar en esas actividades, junto con las partes interesadas y las autoridades competentes.

-.En tercer lugar, la Comisión seguirá vigilando la evolución del mercado en lo que respecta al acceso de los clientes a las ofertas del mercado único, también de cara a la futura aplicación de otras medidas de la Estrategia para el Mercado Único Digital relacionadas con el comercio electrónico.

Finalmente nos atrevemos a adherirnos a una propuesta adelantada por algunos autores $^{25}$ : crear un grupo de trabajo para reflexionar sobre la posibilidad de adoptar

\footnotetext{
${ }^{24}$ Por ejemplo, la crisis de la COVID-19 afectó de manera inmediata al sector audiovisual y es probable que también haya tenido efectos diferidos y más profundos, como señaló el Observatorio Europeo del Sector Audiovisual, que empezó a hacer un seguimiento de las medidas de apoyo a este respecto, véase el siguiente enlace: https://rm.coe.int/the-european-audiovisual-industry-in-the-time-of-covid19/16809ec9cb. Con respecto a los efectos inmediatos, la Comisión intervino con un Marco Temporal relativo a las medidas de ayuda estatal, que ha permitido a los Estados miembros adoptar una serie de medidas de apoyo y medidas sectoriales específicas en el marco del programa Europa Creativa, a saber, la flexibilidad introducida en las medidas de fomento del desarrollo de la industria audiovisual y una adaptación del Instrumento de Garantía de los Sectores Cultural y Creativo que permite una mayor flexibilidad en el reembolso de los préstamos y alienta a las instituciones financieras a prestar más fondos a los sectores cultural y creativo. Además, la Comisión adoptó un plan de recuperación para Europa sin precedentes con el fin de solucionar problemas y prepararse para la próxima generación, que reconoce que las industrias cultural y creativa, incluidos los medios de comunicación y los servicios audiovisuales, forman un importante ecosistema industrial.

25 Véase, por ejemplo: A. MAYORAL (2020). Op. cit., 19-20.
} 
una Directiva o Reglamento para extender la prohibición de los obstáculos a la discriminación debidos al bloqueo geográfico de los clientes que compran productos o servicios en el ámbito territorial de cada Estado miembro... iAunque ello suponga un complejo (¿e insoluble?) conflicto en el marco del inestable equilibrio de competencias entre la UE y dichos Estados!

\section{Bibliografía}

FLÓREZ ROJAS, M. L., «Are online consumers protected from geo-blocking practices within the European Union?», International Journal of Law and Information Technology, 2018, 119-141.

MAYORAL, A., «La visibilidad de la legislación: alcance e intereses en juego», BoDiAlCo, no. 38, 2020, 11-20.

MAZZIOTTI, G., «Is GeoBlocking a Real Cause for Concern in Europe?», EUI Department of Law Research Paper, No. 2015/43, 23 pp, disponible en: https://papers.ssrn.com/sol3/papers.cfm?abstract_id $=2728675$ 